\title{
Belphégor
}

Littérature populaire et culture médiatique

$17 \mid 2019$

Mutations des légitimités dans les productions culturelles contemporaines

\section{Interpréter la pop mainstream ? Critique musicale et légitimation}

\section{Thomas Mercier-Bellevue}

\section{(2) OpenEdition \\ Journals}

Electronic version

URL: https://journals.openedition.org/belphegor/1617

DOI: $10.4000 /$ belphegor.1617

ISSN: 1499-7185

Publisher

LPCM

Electronic reference

Thomas Mercier-Bellevue, "Interpréter la pop mainstream ? Critique musicale et légitimation",

Belphégor [Online], 17 | 2019, Online since 18 April 2019, connection on 25 Auqust 2021. URL: http:// journals.openedition.org/belphegor/1617 ; DOI: https://doi.org/10.4000/belphegor.1617

This text was automatically generated on 25 August 2021.

\section{(c)}

Belphégor est mis à disposition selon les termes de la Licence Creative Commons Attribution - Pas d'Utilisation Commerciale - Pas de Modification 4.0 International. 


\title{
Interpréter la pop mainstream? Critique musicale et légitimation
}

\author{
Thomas Mercier-Bellevue
}

\section{La pop mainstream face à la critique d'art}

1 Y a-t-il une critique musicale consacrée à la pop mainstream ? Si les discours consacrés à ce genre musical sont légion, peut-on qualifier ces derniers de critiques? Il est possible de dresser un constat selon lequel les chansons de pop mainstream ne font que rarement l'objet d'un traitement adéquat par la critique musicale.

2 On peut définir la critique d'art comme un genre littéraire qui vise à décrire, interpréter et évaluer les œuvres d'art qui lui sont contemporaines, ce sur la base de critères explicites et justifiables ${ }^{1}$. Le lieu privilégié de la critique d'art est la presse généraliste ou spécialisée. La critique d'art se distingue de la promotion par son indépendance à l'égard du producteur, de l'histoire de l'art par la contemporanéité de ses objets d'étude, de la théorie de l'art en ce qu'elle prend pour objets des œuvres d'art singulières.

Dans un second temps, on peut définir la pop mainstream comme un genre musical dont les œuvres sont produites par et pour des technologies de diffusion de masse, et dont la production est conçue dans le but d'une diffusion et d'une rentabilité maximisées. Toutefois, la pop mainstream n'est pas uniquement caractérisée par ses modalités de diffusion et par le public visé, mais aussi par ses propriétés stylistiques (récurrence d'un petit nombre de plans formels et de structures harmoniques, accent mis sur le rythme, les arrangements et la mélodie beaucoup plus que sur les paroles et l'harmonie), génétiques (composition collective, prépondérance de la figure du producteur au détriment de celle l'auteur-compositeur), idéologiques (mise à distance des revendications d'authenticité, de subversion et de territorialisation, pleine assomption du rôle de divertissement ${ }^{2}$ ) et pragmatiques (valorisation d'une écoute fragmentaire, réponse somatique ${ }^{3}$ ). 
la grande diversité qu'abrite la pop mainstream, ce qui nous autorise à soutenir qu'il y a davantage de continuités entre Sean Paul et Taylor Swift qu'entre Sean Paul et Capleton.

5 La musique mainstream doit donc être nettement distinguée des musiques folkloriques (le gospel, la forró, le dengaku, etc.) d'une part, et d'autre part des musiques dites indépendantes (le rock, le métal, le hip-hop, la vaporwave, etc.).

6 Une fois nos objets ainsi définis, on peut constater que la pop mainstream, contrairement à d'autres genres musicaux comme le rock, le jazz ou la techno, ne fait que rarement l'objet d'un traitement véritablement critique. La pop mainstream ne dispose pas d'organes critiques qui lui seraient propres (par exemple de revues spécialisées), et quand elles n'ignorent pas simplement les productions appartenant à ce genre musical, les revues généralistes les évoquent selon une méthode et un lexique dont nous allons tenter de montrer l'inadaptation.

7 Il est possible de distinguer quatre attitudes discursives à l'égard de la pop mainstream, attitudes qu'il est, selon nous, impossible de qualifier de critiques si l'on s'en tient à la définition proposée.

On peut d'abord identifier un certain silence de la critique musicale : nombre d'albums pop importants sont simplement tus par les revues de critique musicale. Ainsi, le magazine Rock \& Folk ne fait aucune mention ni de ...Baby One More Time de Britney Spears (1999), ni de Pop Life de David Guetta (2007) ${ }^{4}$. De même, Rolling Stone France et Les Inrocks passent Pop Life sous silence. Sortir de ce mutisme pour évoquer une artiste mainstream peut parfois relever de l'hérésie pour certaines revues, comme en témoigne ce journaliste forcé de se justifier : «On vous entend d'ici : « mais $\mathrm{p}^{* * * * *}$, pourquoi nous parlez-vous de Rihanna????» Parce que, qu'on le veuille ou non, Rihanna fait désormais partie de tout ce qu'on peut englober sous la notion de « pop culture ». $»^{5}$

Dans un second temps, on constate à l'égard de certains artistes de pop mainstream une nette récurrence de l'insulte. Bien qu'une relative vivacité verbale de la critique soit parfois bienvenue, il s'agit selon nous d'une attitude qui, si elle se substitue totalement à l'exigence d'explicitation de ses critères esthétiques, ne relève pas de la critique d'art, d'autant moins quand l'insulte est dirigée ad hominem contre l'artiste et non pas adressée à ses œuvres en guise de critique négative fondée. Le premier album de Britney Spears est ainsi comparé aux " grognements d'une allumeuse pré-pubère " ${ }^{6}$, et David Guetta se voit qualifié d'« apprenti plagiste et tombeur de cagoles à St Tropez, à l'intelligence avoisinant les QI cumulés d'une feutrine de MK2 et d'une serviette éponge Sergio Tacchini $»^{7}$.

Dans un troisième temps, il est possible d'identifier des discours portant sur la pop mainstream, mais qui relèvent davantage du journalisme musical que de la critique. Il s'agit de discours qui mettent de côté les caractéristiques artistiques de la pop pour se concentrer sur son caractère de fait de société. Ces textes insistent sur la dimension personnelle et biographique du fait musical: les goûts de l'artiste, son passé, ses engagements, ses amours... Ce type de discours a tendance à se substituer à la critique musicale proprement dite en minorant, voire en ignorant, la production artistique de la chanteuse ou du chanteur en question. Par exemple, en 1999, Rolling Stone consacre un article de huit pages à la vie intime de Britney Spears ${ }^{8}$ alors que son album n'avait fait l'objet que d'un entrefilet de quelques lignes dans un numéro antérieur ${ }^{9}$. Bien que dans

Belphégor, 17| 2019 
la pop, biographie et musique soient intimement liées, cette attitude discursive ne fait, selon nous, pas office de critique d'art, dans la mesure où ses objets sont des personnes biographiques et non pas des productions artistiques.

11 Enfin, nombre de discours considèrent la pop mainstream dans son ensemble, comme s'il s'agissait d'un objet unifié et homogène. La critique tend ainsi à postuler l'homogénéité d'un genre ou sous-genre musical, et à s'ériger contre celui-ci dans son entièreté, le discréditant au singulier sans distinguer le bon grain de l'ivraie en son sein. Ainsi, certains critiques rock des années 1970 et 1980 ou de bien des critiques techno actuels identifient respectivement «le disco » et «l'EDM ${ }^{10}$ comme des genres musicaux mineurs. Ainsi un article de Slate affirme-t-il que «ce qui est nul, c'est l'EDM $»^{11}$, rejetant en bloc non seulement le genre musical mais aussi la culture festivalière qui lui est inhérente. Un tel postulat d'unité dans la médiocrité, qui n'envisage pas l'éventuelle existence de productions de qualité au sein du genre, ne répond pas à ce que nous posions plus haut comme l'un des réquisits de la critique d'art, à savoir l'attention portée à la singularité des œuvres (il en irait de même avec un postulat d'unité dans la génialité).

Nous voyons donc que la pop mainstream est soit simplement ignorée par la critique musicale, soit traitée d'une manière qu'on peut qualifier de non-critique. Chaque revue considère évidemment la pop mainstream selon sa propre culture éditoriale (on remarque que la critique française est beaucoup plus encline au silence que la critique américaine), mais on peut affirmer que la pop n'est pas prise en considération en tant qu'œuvre d'art. Si elle est évoquée, le traitement qui lui est réservé est celui d'une curiosité, d'un produit dont il faut comprendre le succès, ou d'une simple machine à danser.

Ce dont souffre la pop mainstream, ce n'est pas tant d'une évaluation négative que d'un défaut de légitimité. Ce manque de légitimité transite par la critique en tant que celle-ci en est à la fois le symptôme et l'un des principes générateurs.

La critique est en effet un opérateur d'artification ${ }^{12}$ essentiel ; elle contribue à octroyer le statut d'œuvre d'art, et par là-même une certaine légitimité. Il est en effet possible de postuler un rapport d'équivalence entre le statut ontologique d'œuvre d'art et la légitimité. Comme l'esquisse Bourdieu, le mode d'être des objets culturels implique « une valeur (i.e. une existence) ». ${ }^{13}$ Par cette équivalence, la pop mainstream, privée de valeur, est par là-même privée d'existence en tant qu'œuvre d'art.

\section{La critique d'art comme opérateur d'artification}

15 Il serait possible de minorer l'impact et la gravité de ce manque de légitimité critique en soulignant que la pop mainstream, en tête des ventes et omniprésente dans nos vies quotidiennes, jouit déjà incontestablement d'une certaine valorisation économique et publique. En effet, la pop mainstream ne connaît ni la crise économique, ni celle de l'industrie du disque; de la concurrence culturelle mondialisée, elle sort clairement victorieuse. D'autre part, il s'agit de loin du genre musical qui touche le plus grand nombre de personnes, et celui dont le public compose le spectre géographique, social et culturel le plus large.

16 Si on reconnaît l'existence et la force de ce régime de valorisation économique et publique, pourquoi devrait-on déplorer un manque de légitimité critique? Ne serait-il 
pas possible de consentir à une pluralité de modalités d'appréciation parallèles, c'est-àdire ne convergeant jamais sur un unique objet? Ne serait-il pas préférable d'accepter que, si les musiques savantes requièrent le jugement d'un appareil critique pour exister, les musiques de masse, quant à elles, ont le public et le profit pour seuls juges? Bref, à chaque domaine de production culturelle ses propres modalités d'appréciation. Tenir une telle position revient selon nous d'une part à confondre les différents modes d'appréciation que sont le goût (subjectif), la valorisation (économique) et la légitimation (socio-institutionnelle), et d'autre part à ignorer que la critique d'art n'est pas qu'un discours qui viendrait se surajouter à des œuvres fonctionnant en-deçà de lui. La critique d'art est aussi une instance qui vise à influencer le monde de l'art, et plus encore qui contribue à générer ce dernier.

17 La critique d'art contribue à assigner à certains objets le statut d'œuvres d'art, et donc à leur assigner une certaine dignité ontologique : une légitimité. Le sociologue Mathieu Béra, rappelant la distinction entre critique d'art et promotion, insiste sur le fait que le traitement critique d'un objet n'est pas axiologiquement neutre, mais qu'il contribue à faire passer cet objet dans la sphère du « culturel ». « Ce que l'on critique, écrit Mathieu Béra, est (ou devient) "culturel” (singulier, exceptionnel) tandis que ce qu'on promeut est (ou devient) “industriel” (commun, général). »"

Il convient de mettre l'accent sur ce devenir industriel ou culturel, car ce qui est en jeu est la légitimation plutôt que la légitimité ; le processus plutôt que l'état de fait. Si l'on suit l'analyse de Mathieu Béra, il faut accepter que la critique d'art ne prend pas seulement acte de légitimités existant en-deçà d'elle, mais qu'elle est aussi et surtout un opérateur de transformation ontologique, un opérateur d'artification.

La "théorie institutionnelle de l'art» développée par George Dickie se confronte pleinement à ce processus d'artification en définissant une œuvre d'art comme « (1) un artefact (2) auquel une ou plusieurs personnes agissant au nom d'une certaine institution sociale (le monde de l'art) ont conféré le statut de candidat à l'appréciation. $»^{15}$ Cette définition permet de penser le fait que le statut d'art d'œuvre serait donné de l'extérieur, et non pas dû aux propriétés intrinsèques de l'objet. Dans cette optique, la dimension institutionnelle n'est pas une donnée seconde qui viendrait se surajouter aux œuvres; elle a un rôle ontogénique pour les œuvres ${ }^{16}$. Or, le monde de l'art n'opère pas cette transfiguration ontologique de manière aveugle. Contrairement à ce qu'affirme Dickie lorsqu'il distingue l'usage classificatoire et l'usage évaluatif du concept d'art ${ }^{17}$, l'institution ne se contente pas de proclamer ou d'adouber des objets, elle est aussi un espace hiérarchisé et hiérarchisant. En affirmant que « toute personne qui se considère elle-même comme membre du monde de l'art en est un de ce seul fait ${ }^{18}$, Dickie minore les hiérarchies inhérentes au monde de l'art; il minore le fait que la transfiguration ontologique opérée par l'institution ne s'effectue pas au hasard mais selon certains critères ${ }^{19}$. Tout ne peut pas accéder à la dignité d'art à moins de satisfaire ces critères ; le « devenir œuvre d'art » d'un objet revêt toujours déjà une assignation de valeur.

Comme le souligne Jean-Marie Schaeffer, "[l'identité des œuvres d'art] est toujours celle d'objets évaluativement "pesés", ce qui signifie que leur identification descriptive est toujours enchâssée dans un espace qualitatif. $»^{20} \mathrm{Si}$ on admet que la hiérarchisation institutionnelle est partie prenante de l'ontologie de l'œuvre, il faut reconnaître que classification et assignation de valeur sont toujours consubstantielles. 
21 Cet espace hiérarchisant a ses organes de discrimination entre ce qui peut ou non entrer en son sein, et ces organes lui sont exclusifs ; c'est-à-dire qu'ils n'ont d'existence que dans l'espace de l'art légitime. Du fait même de cette exclusivité, la pop mainstream se trouve dépourvue d'instances de légitimation propres, par opposition à la musique savante, mais aussi par opposition aux musiques indépendantes, qui bénéficient d'instances analogues ${ }^{21}$. Du fait de cette absence de critique, l'artification ne s'opère simplement pas (on pourrait aller jusqu'à dire que la pop mainstream est privée de monde de l'art, au sens de Danto). Seules les œuvres légitimes, c'est-à-dire s'étant vu assigner le statut ontologique d'art et la dignité axiologique inhérente à ce statut, font l'objet d'un traitement critique (la critique étant l'une, sinon la principale, des instances de légitimation). En tant qu'espace hiérarchisé et hiérarchisant, le monde de l'art (dont la critique est partie prenante) a pour effet non seulement de désigner des objets comme œuvres d'art, mais aussi et surtout de tracer les limites de l'art légitime.

22 A la fois symptôme et opérateur de ce processus, la critique se trouve au cœur de la logique de discrédit jetée sur la pop mainstream. Comme nous allons tenter de le montrer, la délégitimation de ce genre musical repose sur le paradigme de la traductibilité herméneutique.

\section{Le paradigme de la traductibilité herméneutique}

23 Le paradigme de la traductibilité herméneutique postule qu'une œuvre d'art est, pour tout ou partie, traduisible par un effort interprétatif, ce qui implique qu'elle n'est pas lisible immédiatement, qu'elle ne permet pas une fréquentation nue, non instruite, sans effort. Si l'herméneutique est la recherche d'un sens caché, l'œuvre d'art serait assimilable à une langue étrangère ou à n'importe quel autre système symbolique qu'il conviendrait de comprendre, dont il faudrait déchiffrer les significations dissimulées.

Si l'on suit Thomas Kuhn, on peut définir un paradigme comme un ensemble de principes et de représentations intériorisées qui norme un domaine du savoir ou de l'action et en assure le fonctionnement régulier. Ainsi, un paradigme scientifique «impose aux scientifiques les règles de leur jeu " $^{22}$; il constitue la «métaphysique courante $»^{23}$ de la science normale. Le paradigme détermine les questions qu'il est possible de poser au sein d'un champ, et les méthodes pertinentes pour y répondre : certaines questions et méthodes sont illégitimes dans le champ, et ce peu importe leur valeur intrinsèque (par exemple, l'astrologie et la divination sont non pertinentes dans le paradigme de la science moderne).

25 Le paradigme de la traductibilité herméneutique agit comme paradigme dans la mesure où il constitue la « métaphysique courante » et non questionnée de la critique d'art : il identifie « de l'extérieur $»^{24}$, depuis l'amont de l'examen critique, ce qui est art et ce qui ne l'est pas, c'est-à-dire ce qui est légitime et ce qui ne l'est pas. Nous souhaitons soutenir l'hypothèse selon laquelle la critique musicale souscrit à ce paradigme, et que c'est cela qui exclut, délégitime la pop mainstream.

L'esthétique contemporaine demeure tributaire d'un modèle que Jean-Marie Schaeffer ${ }^{25}$ identifie comme "romantique ${ }^{26}$. Ce modèle induit des hiérarchies attentionnelles particulières, c'est-à-dire qu'il indexe sa définition de l'art sur un certain nombre de catégorisations évaluatives, de critères fixés déductivement par la philosophie. Ainsi, « les pratiques non conformes à ce qui [est] censé être la tâche de l'heure [sont] tout 
simplement délégitimées en tant qu'art et versées dans le champ de la "décoration" ou de la répétition stérile $»^{27}$. Quels sont ces critères d'exclusion et quelles sont ces tâches assignées à l'art par le modèle romantique ? Si l'on suit l'analyse de J.-M. Schaeffer, le modèle romantique assigne à l'art la tâche de révéler symboliquement des vérités métaphysiques ou religieuses inaccessibles à l'analyse et au discours conceptuels. Etendant à tous les arts le modèle de la poésie, ce modèle soumet l'art à une injonction à dire, à signifier. Le critère d'évaluation de l'œuvre devient la profondeur de son message, et son critère de légitimation devient sa capacité à donner lieu au discours de décryptage visant à faire émerger ce message.

Le paradigme de la traductibilité herméneutique exclut du champ toute œuvre qui ne se prête pas à un travail de décryptage. Une méthode, une modalité possible de fréquentation des œuvres (l'herméneutique), se trouve transformée en critère de légitimité : si une œuvre est réticente à l'analyse interprétative, elle est illégitime. La fin, la dignité et la valeur d'une œuvre d'art résideraient dans le fait de donner lieu à l'interprétation; et inversement une œuvre dont il n'y a rien à dire, une œuvre lisse, qui ne donne pas prise au discours herméneutique, relèverait du non-art. "Dès lors qu'on postule que la traductibilité herméneutique définit l'art, alors toute œuvre qui n'est pas traduisible sera marginalisée ou exclue. $»^{28}$

La critique d'art, dans la mesure où elle vise à interpréter les œuvres, est le lieu privilégié de la traductibilité herméneutique, ce qui nous ramène à ce que nous disions plus haut en identifiant la critique d'art comme l'un des principaux opérateurs d'artification et de légitimation. Une œuvre qui parait intraduisible, et donc qui semble ne pas pouvoir faire l'objet d'un traitement herméneutique par la critique d'art, sera donc exclue du champ de l'art. Ainsi, l'absence de critique qui s'exerce sur la pop mainstream n'est pas axiologiquement neutre : elle est le symptôme du paradigme de la traductibilité herméneutique.

Plus encore, on constate un glissement entre légitimation et évaluation qui fait qu'une œuvre délégitimée se trouve qualifiée comme œuvre de moindre valeur: l'ontologie que charrie le paradigme de la traductibilité herméneutique se trouve érigée en critère d'évaluation. Ne pas donner lieu au travail herméneutique, résister à cet impératif de traductibilité, cela serait le signe d'un défaut de valeur : une œuvre dont il n'y a rien à dire souffrirait d'un manque de profondeur, et donc serait une mauvaise cuvre. Du fait de ce glissement entre légitimation et évaluation, la pop mainstream oscille entre une qualification comme non-art et une qualification comme mauvais art.

Cette structure est particulièrement visible dans l'œuvre d'Arthur Danto. Parce qu'il articule explicitement ontologie de l'œuvre d'art, théorie de l'interprétation et pratique en tant que critique d'art, le travail de Danto est éminemment révélateur des ramifications contemporaines du paradigme de la traductibilité herméneutique. Le travail de critique d'art de Danto pour le magazine The Nation est en effet une émanation directe du grand projet de définition du concept d'art mené en 1981 dans $L a$ Transfiguration du Banal. Danto y propose une définition du concept d'art, censée subsumer l'intégralité des pratiques artistiques. Il aboutit à l'idée selon laquelle l'œuvre d'art est une signification incarnée, c'est-à-dire un système sémantique, dont la modalité symbolique est celle de la métaphore. Ainsi, l'œuvre d'art n'est pas prioritairement un objet destiné à provoquer une réaction sensorielle (une expérience esthétique); «le noyau de [l'œuvre d'art] consiste dans une structure de sens que l'esprit doit saisir. $»^{29}$ Subséquemment, Danto fonde une théorie de la réception 
esthétique comme lecture ou comme décryptage. «La réaction esthétique, affirme-t-il, passe par une médiation conceptuelle $»^{30}$. Ainsi l'expérience esthétique ne peut advenir qu'au terme d'un travail de décodage du système métaphorique qu'est l'œuvre. D'après la théorie dantienne, fréquenter une œuvre d'art, c'est déjà se livrer à un travail critique. La méthodologie de la critique d'art proposée par Arthur Danto, en continuité avec sa théorie de la réception, repose donc sur le postulat selon lequel « les œuvres d'art ne fournissent pas elles-mêmes les éléments nécessaires à leur compréhension " ${ }^{31}$. Dans cette perspective, le critique d'art a pour tâche de « mettre en lumière ce à propos de quoi sont les œuvres - ce qu'elles signifient $»^{32}$, de «mettre au jour la philosophie de l'œuvre $»^{33}$. Le modèle esthétique et critique promu par Danto est donc éminemment intellectualiste et pleinement ancré dans le paradigme de la traductibilité herméneutique. Dans le même mouvement, ce modèle reconduit ce qui nous identifiions plus haut comme le régime de légitimation et d'évaluation induit par le paradigme de la traductibilité herméneutique : «les grandes œuvres d'art, écrit Danto, sont celles qui expriment les pensées les plus profondes $»^{34}$. Danto se situe dans la tradition d'une certaine pratique artistique, à savoir l'art conceptuel, et prétend généraliser les caractéristiques de cette tradition à l'ensemble de l'art. Il découle de cela une délégitimation des œuvres qui n'expriment pas de "pensées profondes ", des œuvres sans aspérités sémantiques, sans chiffre, sans code. De telles œuvres n'ont pas leur place dans la critique d'art. Comme Danto l'affirme lui-même, il ne traitera que d'œuvre « de qualité suffisante pour qu'il ne [lui] reste plus qu'à expliquer la manière dont les œuvres incarnent leur signification $»^{35}$. On voit ici opérer le glissement entre évaluation et légitimation: les œuvres «de qualité " ne sont que les œuvres critiquables, traductibles, et inversement une œuvre qui ne se prête pas à l'interprétation se voit privée de qualité. Il n'y a pas lieu de parler des œuvres dont il n'y a rien à dire, œuvres illégitimées par la prédominance du paradigme de la traductibilité herméneutique qui porte sur elles un jugement de valeur.

31 Il convient maintenant de nous demander pourquoi le paradigme de la traductibilité herméneutique contribue à rendre les œuvres de pop mainstream invisibles, illégitimes ? Ou plutôt, pourquoi la pop mainstream n'intègre-t-elle pas ce paradigme? Il est possible d'affirmer que, de fait, certaines caractéristiques de ces œuvres présentent une résistance à la pratique de l'interprétation.

\section{Interpréter la pop mainstream ?}

32 La pop mainstream contrevient à la vision "romantique » de l'art, dont Jean-Marie Schaeffer montre comment elle est intrinsèquement liée au paradigme de la traductibilité herméneutique. Cette vision repose notamment sur deux présupposés, deux « catégorisations évaluatives $»^{36}$ qui contribuent à délégitimer la pop mainstream. Ces deux présupposés, tributaires de l'héritage kantien, tiennent lieu à la fois de critères définitionnels et de critères d'évaluation. Il s'agit d'une part de l'originalité, d'autre part de l'auctorialité.

33 Il est possible d'affirmer que ces deux éléments sont étrangers aux modalités de production de la pop. Originale, la pop mainstream ne l'est guère, répétant inlassablement des recettes ayant fait leurs preuves suivant l'inusable principe selon lequel «ce qui a plu plaira». De nombreux producteurs avouent ainsi utiliser le « hit predictor », outil qui compare une chanson aux tubes précédents pour déterminer ses 
chances de devenir elle-même un succès. Les chansons de pop sont ainsi aisément comparables à des "produits manufacturés $»^{37}$. De même, l'auctorialité et la figure de l'artiste génial sont largement mises à distance dans le processus de production des hits. «Le processus de création de chansons, constate John Seabrook, s'apparente à une chaîne d'assemblage. Les différentes parties de la chanson peuvent être attribuées à différents spécialistes - auteurs de couplets, forgeurs de hooks, créateurs de ponts, paroliers. ${ }^{38}$ Dans ce processus collectif, et même si certains producteurs pourraient prétendre occuper la place laissée vacante par la figure de l'auteur-compositeurinterprète $^{39}$, l'idée que l'œuvre d'art serait le fruit de la vision personnelle et idiosyncrasique d'un artiste démiurgique s'estompe très largement.

Or, auctorialité et originalité sont deux des présupposés sur lesquels se fondent la pratique herméneutique. Si l'on considère qu'il faut un message, et donc un locuteur, pour se livrer à un travail exégétique, alors l'auctorialité serait en effet une condition nécessaire de l'interprétation. Pour Danto, l'œuvre d'art est à considérer comme un «message émanant de [l'artiste] $»^{40}$, et la tâche du récepteur critique est de traduire et d'expliciter ce message. L'existence, au moins supposée, d'un locuteur singulier est donc nécessaire à tout travail critique sur une œuvre. Corollairement, seuls des messages singuliers et donc originaux seraient à même de donner lieu à un travail interprétatif. Pour que l'interprétation soit singulière il faudrait que l'œuvre le soit aussi. Il semblerait donc que les modalités de production et de locution propre à la pop constituent des résistances à l'interprétation.

Contrairement au rock, qui a largement intériorisé les dynamiques romantiques et modernistes en revendiquant les valeurs d'auctorialité et d'originalité, et dont les critiques ont «utilisé toute la panoplie du romantisme (génie, transcendance, effets corrupteurs du commerce et de la vie de famille ${ }^{41}$ )», la pop mainstream, sans auteur clairement assigné, et ne se souciant guère d'innovation formelle, semble donc impossible à interpréter, indicible, ineffable, d'où son manque de légitimité critique et sa dévaluation au sein du paradigme herméneutique.

Celui-ci assigne à cette dernière l'idée d'une fréquentation nue, immédiate, somatique, l'identifiant à une simple machine à danser, rétive au discours, par opposition à un art légitime dont l'expérience serait nécessairement instruite et médiatisée.

Comme nous le proposions plus haut, il serait possible de s'en tenir là, d'assumer que la pop mainstream est fondamentalement a-critique et que sa capacité à vendre et à faire danser doit être son seul juge. Mais il nous semble nécessaire de réaffirmer la nécessité de la critique pour tout monde de l'art. Comme l'affirme Rainer Rochlitz, «rien n'est plus néfaste, pour l'art, que l'absence d'une critique exigeante $»^{42}$. La nécessité de cette critique est double : il s'agit d'une part d'une nécessité théorique. En effet, comme nous l'avons souligné, il est insuffisant de juger de la valeur d'une catégorie, d'un genre musical, sans juger de la valeur de ses occurrences singulières. Il importe, au sein de la pop mainstream, de distinguer le bon grain de l'ivraie; or le paradigme de la traductibilité herméneutique tend à jeter le silo entier. Cette nécessité de la critique est ensuite pragmatique : une critique évaluative serait bénéfique pour la pop elle-même, dans une perspective d'amélioration du genre musical. Il semble que l'indifférence à l'égard de la valeur des occurrences singulières du champ soit un frein à l'amélioration. Comme le souligne Richard Shusterman, la critique constitue "la surveillance esthétique qui aurait pu rendre l'art populaire meilleur et plus raffiné $»^{43}$. Une critique exigeante serait à même de contrebalancer le jugement économique; elle permettrait 
de ne plus abandonner la pop au seul discours promotionnel, et donc d'encourager l'émergence (ou du moins la prolifération) d'une pop de qualité. Ce que Shusterman propose, ce n'est pas uniquement une conversion du regard, qui par le processus d'artification permis par la critique, amènerait les spectateurs à considérer dans la pop des qualités proprement artistiques. Ce qu'il appelle de ses vœux, c'est une amélioration objective par l'intermédiaire de la critique : artifiée et donc légitimée, la pop aspirerait davantage à être art. Si légitimation et évaluation sont bien liées, légitimer la musique populaire amènerait cette dernière à gagner en qualité.

Mais en disant cela, nous n'avons encore que posé une pétition de principe : comment réaliser cette espérance en la critique alors que nous avons dit que la pop mainstream était peut-être rétive à la pratique de l'interprétation ? Par ailleurs, ce retour de la critique est-il envisageable en-dehors ou en-deçà du paradigme de la traductibilité herméneutique? Ne reviendrait-il pas à intellectualiser artificiellement un genre musical qui ne s'y prête pas?

\section{Détachement de l'œuvre et problème de l'unité de l'art}

Pour répondre à ces questions, nous souhaiterions conclure en mettant l'accent sur l'un des fondements du paradigme de la traductibilité herméneutique et de son corollaire romantique. Cette croyance est double : il s'agit d'une croyance en l'unité du concept d'art, doublée d'une croyance en ce que de l'unité de l'art doit découler une unité de la critique et de ses méthodes.

Cette croyance est, selon nous, fausse et délétère. C'est bien l'exportation du modèle littéraire sur lequel est fondé le paradigme de la traductibilité herméneutique vers les autres arts qui a contribué à délégitimer et dévaluer la pop mainstream. Le retour de la critique que nous appelons de nos vœux doit être une critique non fondée sur ce paradigme, une critique défaite de ses présupposés. Peut-être faut-il accepter que la critique doive s'adapter à son objet ; qu'une critique pop ne doit pas tenter de comparer l'incomparable, d'évaluer Rihanna à l'aune de Schubert (ni même d'ailleurs à l'aune des Beatles), mais qu'il lui revient de fonder sa pratique sur des critères endogènes, adéquats à son objet. Si Richard Shusterman, notamment dans le chapitre de L'art à l'état vif consacré au rap, adopte une stratégie consistant à réduire l'écart entre arts populaires et arts savants, nous pensons plus judicieux d'assumer pleinement cet écart, et d'y circuler en s'interrogeant sur la singularité du genre. Oui, les paroles sont superficielles, les harmonies sont simples, le champ est standardisé ; mais il s'agit de prendre en considération ces caractéristiques non pas comme des défauts ou des manques (manque de profondeur, de complexité, d'originalité), mais comme les caractéristiques propres d'une identité esthétique. Nous partageons ici pleinement la revendication portée par Agnès Gayraud, selon laquelle

l'inauthenticité sous condition de laquelle la pop est née et persiste à vivre n'est rien d'autre que le point d'achoppement de sa vérité esthétique et éthique. Son inauthenticité fondamentale n'est pas ce qui la disqualifie au regard de l'art mais ce qui fait d'elle un art distinct, dont les enjeux et les exigences sont étrangers à la plupart de ceux qui gouvernent l'histoire des musiques savantes. ${ }^{44}$

L'existence d'arts "distincts» dont les exigences sont parfaitement singulières contrevient à un désir d'unité infondé : tenter de saisir la « vérité esthétique » d'un art 
à l'aune de celle d'un autre est une tentative vouée à l'échec. Les différentes pratiques de création et de réception s'épanouissent selon leurs propres logiques et leurs propres critères, et cette diversité est certainement irréductible. Par conséquent, la critique d'art elle-même doit être irréductiblement diverse, moduler ses critères d'appréciation, d'examen et d'évaluation en fonction des spécificités de son objet. « Différentes œuvres d'art sont, ou peuvent être, blâmables ou louables pour différentes raisons, et pas toujours pour les mêmes raisons. Une propriété peut être louée dans une peinture et blâmée dans une autre. $»^{45}$ Dans cette perspective, le fait que la pop mainstream échappe ou résiste à la pratique exégétique devrait être une caractéristique singulière à prendre en compte plutôt que le promontoire d'une stratégie d'illégitimation.

Chaque art appelle son propre lexique critique et ses propres critères d'appréciation, et ce dans la mesure où chaque pratique artistique ne se réduit pas à un ensemble d'œuvres d'art autonomes, mais charrie avec elle un ensemble singulier de pratiques (de création et de réception) et de représentations. Chaque champ artistique s'apparente à un jeu d'accords et de médiations entre artistes, institutions et spectateurs, et ces jeux sont potentiellement hétérogènes entre eux, d'où la nécessité de passer d'une réflexion sur le jugement critique en général à la possibilité d'une "pluralité de connaisseurs" agissant dans «des domaines d'appréciations différents. ${ }^{46}$ Ces jeux différents sont potentiellement hétérogènes, intraduisibles entre eux; d'où le fait que "l'expertise interne à un jeu de langage n'a pas à être exportée en dehors de ce jeu $»^{47}$. L'expertise en question ne doit pas séparer l'œuvre de ses ramifications culturelles, car «se représenter un jeu de langage veut dire se représenter une forme de vie. $»^{48}$ De même que Wittgenstein refuse qu'on réduise le langage à la dénotation, nous refusons de réduire la pop à un ensemble de chansons. Ces chansons en effet ne peuvent se comprendre sans la "forme de vie ", c'est-à-dire l'ensemble de pratiques, qui les accompagnent. D'une pratique artistique à l'autre, ce ne sont pas que les formes qui changent ; «le jeu tout entier est différent $»^{49}$, et ce dans la mesure où « c'est une culture tout entière qui ressortit à un jeu de langage » ${ }^{50}$.

Ainsi faut-il comprendre tout d'abord que la pop mainstream n'est pas réductible à des chansons détachées de tout un jeu, jeu de création (la composition collective), jeu de réception (la culture du club), de réappropriation (les remix). La pop mainstream ne se comprend que comme art total, engageant non seulement des éléments musicaux, mais aussi des éléments visuels, chorégraphiques et narratifs ${ }^{51}$. La critique musicale se fourvoie en aspirant à couler le mainstream dans le moule d'une interprétation fondée sur l'idée de transcendance des œuvres d'art, c'est-à-dire de détachement des œuvres à l'égard de leurs modalités concrètes de production et de réception. Elle se fourvoie également en méconnaissant le fait que la pop mainstream est quasiment incommensurable aux musiques indépendantes, tributaires d'une idéologie moderniste valorisant l'auctorialité, la virtuosité, l'authenticité, et relevant de stratégies d'écoute différentes; la forme de vie toute entière est différente. Il serait réducteur de n'envisager la pop mainstream que comme une stratégie de communication (est mainstream ce qui vise et atteint le très grand public), car cette stratégie engage en profondeur l'ensemble des caractères (esthétiques, génétiques, pragmatiques et idéologiques) des œuvres. Il ne s'agit là que de pistes, qui feront l'objet d'un texte à venir, mais la légitimation de la pop mainstream ne peut pas faire l'économie d'une critique qui prenne en compte ces éléments, qui lui sont inhérents. 


\section{Conclusion}

Dans ce texte, nous avons tenté d'expliquer le discrédit dont fait l'objet la pop mainstream en nous appuyant sur les effets de la critique d'art. Si devenir une œuvre d'art, c'est toujours entrer dans cet espace hiérarchisant et légitimant qu'est le monde de l'art, alors il faut reconnaître que les instances qui structurent cet espace, et notamment la critique d'art, sont des opérateurs de légitimation. Nous avons montré que la légitimation s'opère selon un ensemble critériologique qu'on peut subsumer comme paradigme de la traductibilité herméneutique, et que la pop mainstream, parce qu'elle résiste à la pratique de l'interprétation, se trouve marginalisée par ce paradigme. Nous maintenons cependant la possibilité et la nécessité d'une critique portant sur ce genre musical, critique qui pour être adéquate à son objet doit abandonner la pratique d'une interprétation purement sémantique pour se diriger vers l'étude et l'évaluation des formes de vie que les œuvres pop charrient avec elles. Enfin, nous devons reconnaître qu'une telle critique, issue des cultural studies, est loin d'être un vœu pieux car elle n'est pas inexistante (ainsi les cultural studies et les visual studies ont-elles popularisé l'analyse de clips et l'analyse des narrativités propres aux icônes de la culture pop). Nous n'avons ici analysé que les fondements d'un mouvement déjà en marche de légitimation critique des musiques populaires, et nous ne pouvons que nous en réjouir.

\section{BIBLIOGRAPHY}

- Mathieu Béra, «Critique d'art et/ou promotion culturelle ? ", Réseaux, 2003/1, nº 117.

- Pierre Bourdieu, «Le marché des biens symboliques », L'Année Sociologique, 22, 3, 1971.

- Arthur C. Danto, « Le monde de l'art» (1964), dans Danielle Lories (éd.), Philosophie analytique et esthétique, Paris, Klincksieck, 2004.

- Arthur C. Danto, La Madone du futur (2000), Paris, Seuil, 2003, tr. fr. Claude Hary-Schaeffer.

- Arthur C. Danto, La Transfiguration du banal (1981), Paris, Seuil, 1989, tr. fr. Claude HarySchaeffer.

- George Dickie, « Définir l'art » (1973), dans Gérard Genette (dir.), Esthétique et poétique, Paris, Seuil, « Points».

- Albert Dresdner, La Genèse de la critique d'art, Paris, Editions de l'ENSBA, 2005.

- Simon Frith, « Retour sur l'esthétique de la musique pop », Rue Descartes, 2006/2, n 60, tr. fr. J.-

P. Penasse et P. Mathias.

- Agnès Gayraud, « Faustus et moi. L'inauthenticité de la pop », Audimat, n 1, 2013.

- Agnès Gayraud, « Doit-on avoir peur de la pop mainstream (et existe-t-elle toujours) ? ", entretien avec Carole Boinet, Les Inrocks, 04/11/2016, en ligne. 
- Nathalie Heinich et Roberta Shapiro, De l'artification. Enquêtes sur le passage à l'art, Paris, 2012, Editions de l'EHESS, « Cas de figure ».

- Emmanuel Kant, Critique de la faculté de juger (1790), § 46, Paris, GF, 1995, tr. fr. A. Renaut.

- William E. Kennick, « Does Traditional Aesthetics Rest on a Mistake ?», Mind, 67, 276, 1958.

- Thomas S. Kuhn, La Structure des révolutions scientifiques (1962/1970), Paris, Seuil, 1982, tr. fr. L. Meyer.

- Yves Michaud, Critères esthétiques et jugement de goût, Nîmes, Editions Jacqueline Chambon, 1999.

- Richard Mèmeteau, Pop Culture. Réflexions sur les industries du rêve et l'invention des identités, Paris, La Découverte, «Zones », 2014.

- Emmanuel Parent, «La question des émotions dans les musiques populaires », in P.-H. Frangne, H. Lacombe, M. Massin, T. Picard (dir.), La valeur de l'émotion musicale, Rennes, PUR, 2017, « Aesthetica ».

- Roger Pouivet, L'œuvre d'art à l'âge de sa mondialisation. Un essai d'ontologie de l'art de masse, Bruxelles, La Lettre Volée, 2003.

- Rainer Rochlitz, L'art au banc d'essai, Paris, Gallimard, « NRF Essais », 1998.

- John Seabrook, Hits ! Enquête sur la fabrique des tubes planétaires, Paris, Philharmonie / La Découverte, 2016.

- Jean-Marie Schaeffer, « Système, histoire et hiérarchie : le paradigme historiciste en théorie de l'art », dans Georges Roque (dir.), Majeur ou mineur ? Les hiérarchies en art, Nîmes, Jacqueline Chambon, 2000.

- Richard Shusterman, L'art à l'état vif. La pensée pragmatiste et l'esthétique populaire, 1991, Paris, Les éditions de minuit, « Le Sens Commun », tr. fr. C. Noille.

- Richard Shusterman, « Légitimer la légitimation de l'art populaire », Politix, 6, 24, 1993.

- Revue Volume!, « Ecoutes », 10-1, décembre 2013.

- Revue Volume!, « Watching Music : culture du clip musical », 14-2, 2017.

- Ludwig Wittgenstein, Recherches Philosophiques, Paris, Gallimard, 2004.

- Ludwig Wittgenstein, « Leçons sur l'esthétique », in Leçons et conversations, Paris, Gallimard, «Folio Essais », 1992, tr. fr. J. Fauve.

\section{NOTES}

1. Nous reprenons ici certains éléments de la définition de la critique d'art proposée par Albert Dresdner dans La Genèse de la critique d'art, Paris, Editions de l'ENSBA, 2005, p. 31.

2. Voir Agnès Gayraud, « Doit-on avoir peur de la pop mainstream (et existe-t-elle toujours)?», entretien avec Carole Boinet, Les Inrocks, 04/11/2016, en ligne.

3. Voir Emmanuel Parent, «La question des émotions dans les musiques populaires », in P.-H. Frangne, H. Lacombe, M. Massin, T. Picard (dir.), La valeur de l'émotion musicale, Rennes, PUR, 2017, « Aesthetica ».

4. Cela serait normal si Rock \& Folk ne traitait que l'actualité du rock et de la folk, ce qui n'est déjà plus le cas aux périodes concernées, puisque les numéros consultés contiennent des articles sur bien d'autres genres musicaux, y compris la house (avec LCD Soudsystem, dans le $n^{\circ} 476$ ) ou le rap léger (avec Zebda dans le $n^{\circ} 377$ ). 
5. «Rihanna, des fans et beaucoup de cris : trois heures d'hystérie », Les Inrocks, 06/06/2014, en ligne.

6. " growling jailbait». Le terme "jailbait», difficilement traduisible, désigne péjorativement une sorte de Lolita entreprenante et très consciente du trouble qu'elle inspire. Rolling Stone, $\mathrm{n}^{\circ}$ 805, février 1999.

7. Christophe Conte, « Cher David Guetta », Les Inrocks, 20/05/2011, en ligne.

8. "Inside the Heart, Mind and Bedroom of a Teen Dream ", Rolling Stone, $n^{\circ}$ 810, avril 1999. Le reportage d'environ huit pages est constitué de plus de six pages de photos très ambiguës mises en scène par le célèbre et sulfureux photographe David Lachapelle.

9. Rolling Stone, $\mathrm{n}^{\circ} 805$, février 1999.

10. Acronyme pour «Electro Dance Music », genre musical grand public hybride entre la house, la pop et la trance, et représenté notamment par David Guetta, Avicii, Steve Aoki ou Mike Posner. 11. Vincent Glad, «Le meilleur DJ du monde est une escroquerie artistique », Trax, 23/10/2015, en ligne.

12. Ce concept a été développé par Nathalie Heinich et Roberta Shapiro, notamment dans l'ouvrage collectif qu'elles ont dirigé : De l'artification. Enquêtes sur le passage à l'art, Paris, 2012, Editions de l'EHESS, « Cas de figure ». Nous le leur empruntons, bien que notre interprétation de l'articulation entre artification et légitimation diverge sensiblement de la leur.

13. Pierre Bourdieu, «Le marché des biens symboliques ", L'Année Sociologique, 22, 3, 1971, p. 59.

14. Mathieu Béra, «Critique d'art et/ou promotion culturelle ? ", Réseaux, 2003/1, n 117, p. 162.

15. George Dickie, «Définir l'art » (1973), dans Gérard Genette (éd.), Esthétique et poétique, Paris, Seuil, « Points », p. 22.

16. Certes, le monde de l'art n'est pas réductible à la critique d'art, mais on ne saurait renier à la critique son rôle proéminent dans le monde en question, dans la fixation de ses codes et dans la délimitation de ses frontières.

17. George Dickie, « Définir l'art », art. cit. p. 13.

18. Ibid., p. 24.

19. Pour ces deux raisons, nous utilisons la locution «monde de l'art " plutôt au sens de Danto qu'au sens de Dickie. Pour Danto le monde de l'art n'est pas uniquement l'ensemble des acteurs et de leurs interactions, mais aussi une « atmosphère de théorie artistique » qui oriente le regard et hiérarchise les possibilités. "Le monde de l'art» (1964), dans Danielle Lories (éd.), Philosophie analytique et esthétique, Paris, Klincksieck, 2004, p. 193.

20. Jean-Marie Schaeffer, "Système, histoire et hiérarchie : le paradigme historiciste en théorie de l'art ", dans Georges Roque (dir.), Majeur ou mineur? Les hiérarchies en art, Nîmes, Jacqueline Chambon, 2000.

21. On songe en premier lieu à la critique rock, qui a contribué à légitimer le genre au point qu'il est aujourd'hui, à l'instar du jazz ou de certains pans de la chanson, largement patrimonialisé. Simon Frith évoque une «critique institutionnalisée [ayant] développé sa propre terminologie esthétique. » (S. Frith, « Retour sur l'esthétique de la musique pop », Rue Descartes, 2008/2, n 60, tr. fr. J.-Ph. Pinasse et P. Mathias, p. 66).

22. Thomas S. Kuhn, La Structure des révolutions scientifiques (1962/1970), Paris, Seuil, 1982, tr. fr. Laure Meyer, p. 66-69.

23. Ibid., p. 38.

24. Ibid., p. 37.

25. Jean-Marie Schaeffer, «Système, histoire et hiérarchie : le paradigme historiciste en théorie de l'art », art. cit.

26. A ce modèle romantique, il faudrait ajouter le modèle moderniste. De ces deux modèles, le paradigme herméneutique est l'un des points de convergence.

27. Ibid., p. 267.

28. Ibid., p. 270. 
29. Arthur C. Danto, La Madone du futur (2000), Paris, Seuil, 2003, tr. fr. Claude Hary-Schaeffer, p. 12.

30. Arthur C. Danto, La Transfiguration du banal (1981), Paris, Seuil, 1989, tr. fr. Claude HarySchaeffer, p. 155.

31. Arthur C. Danto, La Madone du futur, op. cit. p. 9.

32. Ibid., p. 10.

33. Ibid., p. 12.

34. Ibid., p. 10.

35. Ibid., p. 13.

36. Jean-Marie Schaeffer, « Système, histoire et hiérarchie », art. cit. p. 268.

37. John Seabrook, Hits! Enquête sur la fabrique des tubes planétaires, Paris, Philharmonie / La Découverte, 2016, p. 14.

38. Ibid., p. 245.

39. La reconnaissance du producteur ne concerne que quelques figures emblématiques, souvent rendues publiques par leur carrière solo parallèle (Dr. Dre, Moroder...). De plus il n'est pas rare que plusieurs producteurs contribuent à un même album (voire à un même morceau), ce qui dilue la possibilité d'identifier l'auteur en eux. Enfin, le producteur est souvent présenté comme une figure artisanale, comme un maitre d'œuvre ou un défricheur de tendances, qui se substitue difficilement à la figure romantique et moderniste de l'artiste.

40. Arthur C. Danto, La Transfiguration du banal, op. cit. p. 100.

41. Simon Frith, « Retour sur l'esthétique de la musique pop », art. cit. p. 66.

42. Rainer Rochlitz, L'art au banc d'essai, Paris, Gallimard, « NRF Essais », 1998, p. 187.

43. Richard Shusterman, « Légitimer la légitimation de l'art populaire », Politix, 6, 24, 1993, p. 156. En ligne.

44. Agnès Gayraud, "Faustus et moi. L'inauthenticité de la pop », Audimat, n 1, 2013, p. 85. Il convient toutefois de souligner qu'Agnès Gayraud parle de la «pop » au sens large de musiques populaires enregistrées, et pas spécifiquement de ce que nous désignons comme «pop mainstream ».

45. William E. Kennick, « Does Traditional Aesthetics Rest on a Mistake ? , Mind, 67, 276, 1958, p. 331.

46. Yves Michaud, Critères esthétiques et jugement de goût, Nîmes, Éditions Jacqueline Chambon, 1999 , p. 25.

47. Ibid., p. 23.

48. Ludwig Wittgenstein, Recherches Philosophiques, Paris, Gallimard, 2004, § 19, p. 35.

49. Wittgenstein, «Leçons sur l'esthétique», § 23 in Leçons et conversations, Paris, Gallimard, «Folio Essais », 1992, tr. fr. Jacques Fauve, p. 27.

50. Ibid., § 26, p. 28.

51. Voir à ce sujet les analyses de Richard Mèmeteau, Pop Culture. Réflexions sur les industries du rêve et l'invention des identités, Paris, La Découverte, «Zones », 2014, introduction et chapitre 1.

\section{ABSTRACTS}

La critique musicale, parce qu'elle est un opérateur essentiel de légitimation, contribue au discrédit dont souffre la pop mainstream. En étudiant le paradigme de la traductibilité 
herméneutique, dont la critique est tributaire, nous souhaitons mettre au jour les racines théoriques du sceau d'illégitimité dont ce genre musical est frappé. Ainsi, nous poserons les bases d'une critique musicale prenant en considération les spécificités de la pop.

INDEX

Mots-clés: Art, culture de masse, hiérarchies culturelles, musiques populaires, réception critique

\section{AUTHOR}

\section{THOMAS MERCIER-BELLEVUE}

Normalien, agrégé de philosophie. Doctorant-contractuel - Paris-Sorbonne Université, Centre Victor Basch (EA 3552) 